\title{
Multisociety statement on coronavirus disease 2019 (COVID-19) vaccination as a condition of employment for healthcare personnel
}

\author{
David J. Weber MD, MPH${ }^{1}$, Jaffar A. Al-Tawfiq MD²,3,4, Hilary M. Babcock MD, MPH ${ }^{5}$, Kristina Bryant MD ${ }^{6}$, Marci Drees MD, \\ $\mathrm{MS}^{7}$, Ramy Elshaboury PharmD ${ }^{8}$, Katharine Essick JD ${ }^{9}$, Mohamad Fakih MD, MPH ${ }^{10}$, David K. Henderson MD $^{11}$, \\ Waleed Javaid MD ${ }^{12}$, Diane Juffras JD, PhD ${ }^{1}$, Robin L.P. Jump MD, PhD ${ }^{13,14}$, Francesca Lee MD ${ }^{15}$, Anurag N. Malani MD ${ }^{16}$, \\ Trini A. Mathew MD, MPH ${ }^{17,18}$, Rekha K. Murthy MD ${ }^{19}$, David Nace MD, MPH ${ }^{20}$, Tara O'Shea BS ${ }^{19}$, Erica Pettigrew MD, JD, \\ $\mathrm{MPH}^{1,21}$, Ann Marie Pettis RN, BSN, CIC ${ }^{22}$, Joshua K. Schaffzin MD, PhD ${ }^{23}$, Erica S. Shenoy MD, PhD ${ }^{8,24}$, \\ Julie Vaishampayan MD, MPH${ }^{25}$, Zanthia Wiley MD ${ }^{26}$, Sharon B. Wright MD, MPH ${ }^{24,27}$, Deborah Yokoe MD, MPH ${ }^{28}$ and \\ Heather Young MD 29
}

${ }^{1}$ University of North Carolina at Chapel Hill, Chapel Hill, North Carolina, ${ }^{2}$ Johns Hopkins Aramco Healthcare, Dhahran, Saudi Arabia, ${ }^{3}$ Indiana University School of Medicine, Indianapolis, Indiana, ${ }^{4}$ Johns Hopkins University School of Medicine, Baltimore, Maryland, ${ }^{5}$ Washington University School of Medicine, St. Louis, Missouri, ${ }^{6}$ University of Louisville, Louisville, Kentucky, ${ }^{7}$ ChristianaCare, Wilmington, Delaware, ${ }^{8}$ Massachusetts General Hospital, Boston, Massachusetts, ${ }^{9}$ University of California, Oakland, California, ${ }^{10}$ Ascension, St. Louis, Missouri, ${ }^{11}$ National Institutes of Health, Bethesda, Maryland, ${ }^{12}$ Icahn School of Medicine at Mount Sinai, New York, New York, ${ }^{13}$ Geriatric Research Education and Clinical Center (GRECC) at the Veterans' Affairs Northeast Ohio Healthcare System, Cleveland, Ohio, ${ }^{14}$ Case Western Reserve University School of Medicine, Cleveland, Ohio, ${ }^{15}$ University of Texas Southwestern, Dallas, Texas, ${ }^{16}$ St. Joseph Mercy Health System, Ann Arbor, Michigan, ${ }^{17}$ Beaumont Hospital, Royal Oak, Michigan, ${ }^{18}$ HealthTAMCycle3, PLLC , Troy, Michigan, ${ }^{19}$ Cedars-Sinai Medical Center, Los Angeles, California, ${ }^{20}$ University of Pittsburgh, Pittsburgh, Pennsylvania, ${ }^{21}$ Orange County Health Department, Chapel Hill, North Carolina, ${ }^{22}$ University of Rochester Medicine, Rochester, New York, ${ }^{23}$ Cincinnati Children's Hospital Medical Center, Cincinnati, Ohio, ${ }^{24}$ Harvard Medical School, Boston, Massachusetts,

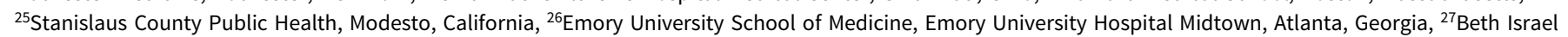
Lahey Health, Burlington, Massachusetts, ${ }^{28}$ University of California San Francisco, San Francisco, California and ${ }^{29}$ Denver Health Medical Center, Denver, Colorado

\section{Executive Summary and Recommendation}

This consensus statement by the Society for Healthcare Epidemiology of America (SHEA) and the Society for PostAcute and Long-Term Care Medicine (AMDA), the Association for Professionals in Epidemiology and Infection Control (APIC), the HIV Medicine Association (HIVMA), the Infectious Diseases Society of America (IDSA), the Pediatric Infectious Diseases Society (PIDS), and the Society of Infectious Diseases Pharmacists (SIDP) recommends that coronavirus disease 2019 (COVID-19) vaccination should be a condition of employment for all healthcare personnel in facilities in the United States. Exemptions from this policy apply to those with medical contraindications to all COVID-19 vaccines available in the United States and other exemptions as specified by federal or state law. The consensus statement also supports COVID-19 vaccination of nonemployees functioning at a healthcare facility (eg, students, contract workers, volunteers, etc). This recommendation is based on several points:

- The COVID-19 vaccines available in the United States under the Food and Drug Administration (FDA) emergency use authorization (EUA) have high efficacy to prevent symptomatic COVID-19, even higher efficacy to prevent serious COVID-19

Author for correspondence: David J. Weber, E-mail: david.weber@unchealth.unc.edu Cite this article: Weber DJ, et al. (2022). Multisociety statement on coronavirus disease 2019 (COVID-19) vaccination as a condition of employment for healthcare personnel. Infection Control \& Hospital Epidemiology, 43: 3-11, https://doi.org/10.1017/ice.2021.322 (ie, hospitalizations and deaths), and high effectiveness against symptomatic and asymptomatic COVID-19.

- The COVID-19 vaccines under an FDA EUA have similar safety profiles to vaccines that are currently fully FDA approved, and they are supported by efficacy trials and effectiveness studies.

- Full vaccination against COVID-19 offers several advantages regarding patient and healthcare personnel (HCP) safety: individual protection against COVID-19; further protection for patients and HCP who are unable to receive COVID-19 vaccination or are not able to mount an adequate immune response; reduced risk of asymptomatic or presymptomatic transmission of severe acute respiratory coronavirus virus 2 (SARS-CoV-2) between HCP, from HCP to patients, or from patients to HCP; reduced risk of transmitting infection to household members and community contacts; and increased protection for the healthcare workforce in the community setting.

- The COVID-19 vaccines appear to retain good effectiveness against currently circulating SARS-CoV-2 variants against symptomatic illness and to have even higher effectiveness against severe disease.

- Prior experience and current information suggest that a sufficient vaccination rate is unlikely to be achieved without making COVID-19 vaccination a condition of employment.

- The statement is consistent with federal law and regulations.

However, some information is not yet known. For example, additional data are needed on the duration of protection provided by COVID-19 vaccines and on the effectiveness of vaccines in 
immunocompromised persons. Data from randomized clinical trials in pregnancy are not yet available, although no maternal or fetal harm has yet been reported, and $>120,000$ pregnant people have received a COVID-19 vaccine. ${ }^{1}$

We have identified medical contraindications and other exemptions as specified by federal and state law that exempt HCP from being required to receive COVID-19 vaccination. Exemptions should be handled within the occupational health program with the engagement of human resources and/or legal departments as appropriate. These exemptions are not contraindications, and healthcare facilities may wish to allow pregnant HCP to postpone receipt of the vaccine until after delivery. Pregnant HCP with questions or concerns about COVID-19 vaccination should be encouraged to speak to their healthcare provider(s) following their pregnancy. Pregnant and lactating HCP should be allowed to receive a vaccine because, as noted by the Centers for Disease Control and Prevention (CDC), "pregnant and recently pregnant people are more likely to get severely ill from COVID-19 compared to nonpregnant people." ${ }^{\prime 2}$ It is important to specify that persons who have had COVID-19 should receive a COVID-19 vaccine as recommended by CDC.

Healthcare facilities should provide an inclusive and transparent process that facilitates and acknowledges input from healthcare personnel and other stakeholders before reaching a decision to adopt a policy of vaccination as a condition of employment. If a healthcare facility decides that requiring a vaccine as a condition of employment is not possible at the present time, the facility should ensure that, following the principles of diversity, equity, and inclusion, it has implemented all methods to improve vaccine coverage described herein, including endorsement by senior leadership, appropriately educating HCP about the vaccine (as required under the EUA), removing financial and physical barriers to access to the vaccine such as providing paid time for vaccination and recovery from vaccine side effects [required by the Occupational Safety and Health Administration (OSHA)], ${ }^{3}$ and providing locations and times convenient for HCP receive it. If minimal adequate coverage (eg, $>90 \%$ based on minimum influenza vaccination rates $^{4}$ ) is not achieved within a reasonable period (eg, 1-3 months), the facility should implement a policy of requiring COVID-19 vaccination as a condition of employment.

\section{Background}

The coronavirus disease 2019 (COVID-19) pandemic has had a profound impact on the United States and across the globe. More than 33 million Americans ( $>1$ in 10) have been documented to have acquired severe acute respiratory coronavirus virus 2 (SARS-CoV-2) infection. ${ }^{5}$ The true percentage of the population that has been infected may never be known with certainty, given the large proportion of undocumented cases, but this number likely greatly exceeds the numbers reported in official accounts. ${ }^{6}$ More than 600,000 Americans have died from the disease. ${ }^{5}$ At the peak of the third wave of the pandemic in the United States, nearly 260,000 cases were reported per day and nearly 160,000 COVID-19 patients were hospitalized in the United States each week. ${ }^{5}$

Historically, the most effective strategies for managing viral illnesses (eg, measles, rubella, and influenza) have been by vaccination, with vaccine manufacturers being required to follow detailed procedures for demonstrating safety and efficacy before applying to the US Food and Drug Administration (FDA) for licensure. ${ }^{7}$ In emergent situations, pharmacotherapies, including vaccines, may be granted emergency use authorization (EUA) by FDA to allow vaccine administration in situations in which no effective alternatives are available. ${ }^{8}$ Reflecting the urgent need, the EUA requires fewer months of safety and efficacy data (typically 2-3 months of experience), whereas full FDA approval requires at least 6 months of data that can be evaluated in detail. In the United States, 3 COVID-19 vaccines have been granted EUAs.

As yet, these vaccines have not received formal FDA approval, although both Pfizer-BioNTech and Moderna filed for formal FDA approval in 2021. ${ }^{9,10}$ According to the World Health Organization (WHO), nearly 100 companies around the world have vaccines in clinical evaluation that have been built on various platforms. ${ }^{11}$ In addition to the mRNA and adenovirus platforms, vaccines using protein subunits, DNA plasmids, and recombinant nanoparticles are currently in clinical trials in the United States.

Vaccines recommended for healthcare personnel (HCP) by the CDC Advisory Committee on Immunization Practices (ACIP) have been offered to HCP for decades. In the last 15 years, increasing numbers of healthcare organizations have instituted a requirement for receipt of ACIP-recommended vaccines for HCP to reliably and sustainably increase HCP vaccination rates. ${ }^{12}$

This consensus statement by the Society for Healthcare Epidemiology of America (SHEA), with AMDA - The Society for Post-Acute and Long-Term Care Medicine, The Association for Professionals in Infection Control and Epidemiology (APIC), the HIV Medicine Association (HIVMA), the Infectious Diseases Society of America (IDSA), the Pediatric Infectious Diseases Society (PIDS), and the Society of Infectious Diseases Pharmacists (SIDP), was reviewed and is endorsed by these organizations. It recommends that COVID-19 vaccination be a condition of employment for all healthcare personnel. Exemptions from this policy apply to those with medical contraindications to all COVID-19 vaccines available in the United States and other exemptions as specified by federal or state law. The consensus statement also supports COVID-19 vaccination of nonemployees functioning at a healthcare facility (eg, students, contract workers, volunteers, etc).

\section{Methods}

The panel that developed this statement is composed of multiorganizational, multidisciplinary experts working in healthcare epidemiology, infection prevention, infectious diseases, pharmacy, public health, law, and human resources. It includes representatives from the SHEA Board of Trustees, the SHEA Guidelines Committee, and other SHEA leaders, as well as organizational representatives from AMDA, APIC, HIVMA, IDSA, PIDS, and SIDP.

The recommendation that COVID-19 vaccination should be a condition of employment for all healthcare personnel, with exemptions applying for those with medical contraindications to all COVID-19 vaccines available in the United States and other exemptions as specified by federal or state law, was reached through a 3-round Delphi process. Consensus was achieved.

\section{Intended use}

This statement is intended for consideration by employers of healthcare personnel and others in the service of health care who work or operate in US healthcare settings. It identifies legal issues that should be considered; the statement is not legal advice. Employers should consult their own attorneys when making decisions regarding the implementation of a policy of COVID-19 vaccination as a condition of employment. Likewise, this statement is not meant to be a substitute for judgment by qualified professionals 
regarding clinical decisions. Finally, the recommendations and views are the authors' and do not necessarily reflect the positions of their affiliated instiutions.

\section{Vaccine efficacy and real-world effectiveness}

\section{Efficacy in clinical trials}

Vaccine efficacy and real-world effectiveness study results and references are summarized in Table 1 in Supplementary material. All 3 COVID-19 vaccines with current FDA EUAs have shown high overall vaccine efficacy by $\geq 14$ days after receipt of the second vaccine dose for mRNA vaccines ${ }^{a}$ or a single dose for the adenovirus vector vaccine ${ }^{b}$ to prevent symptomatic COVID-19 infection in phases 3 and 4 of randomized placebo-controlled trials $(94.1 \%-$ $95 \%$ for the 2 mRNA vaccines and $66.9 \%$ for the adenovirus vector vaccine). ${ }^{13,14}$ Similar vaccine efficacy has been observed across subgroups based on age, gender, race or ethnicity, and coexisting medical conditions.

\section{Real-world effectiveness}

The growing number of real-world studies show similar overall vaccine effectiveness for preventing symptomatic and asymptomatic COVID-19. Studies assessing immunogenicity and limited vaccine effectiveness data suggest that vaccine effectiveness is likely lower for immunocompromised persons. ${ }^{15-17}$ All FDA EUA vaccines have demonstrated comparable effectiveness against the alpha variant of SARS-CoV-2 (B.1.1.7), and data continue to evolve around other circulating variants. Several studies have demonstrated lower SARS-CoV-2 viral loads in nasal specimens among fully vaccinated persons compared to unvaccinated persons who develop COVID-19. ${ }^{18,19}$ These findings, among other real-world data, suggest that vaccination reduces the likelihood of COVID19 transmission from infected persons to their contacts, including household contacts. ${ }^{20-22}$

\section{Boosters}

Given the short duration of immunity to seasonal coronaviruses after infection ${ }^{23}$ and the speed with which disparate viral variants with mutated spike proteins are emerging, at some point in the future, a booster may be required for waning immunity and/or improved coverage for emerging variants. ${ }^{24-26}$

\section{COVID-19 vaccine safety}

\section{Clinical trial data}

Although the pace of COVID-19 vaccine development has been unprecedented, the 3 vaccines currently available in the United States were authorized after randomized controlled trials ${ }^{27-29}$ as large as or larger than those undertaken for prior vaccines (30,000-44,000 participants per trial, randomized 1:1 vaccine vs placebo), with diverse participants in terms of age, race, ethnicity, and comorbid conditions. These trials demonstrated their efficacy

\footnotetext{
aThe mRNA vaccines require 2 doses (21 days apart for Pfizer-BioNTech and 28 days apart for Moderna). ${ }^{7,8}$ After injection of mRNA vaccines, host cells utilize the vaccine mRNA that contains the genetic code for the viral spike protein to produce either all or a large part of the SARS-CoV-2 spike protein, to which the body then responds immunologically. ${ }^{27,28}$

${ }^{\mathrm{b}}$ The Johnson \& Johnson (J\&J)/Janssen vaccine requires a single dose. The adenovirus vector vaccines work by using a non-replicating adenovirus that has been altered to include the gene that codes for the spike protein. The nonreplicating adenovirus enters host cells and triggers the production of the SARS-CoV-2 spike protein, ${ }^{14,15}$ to which the body responds.
}

and safety. The 3 vaccines had similar rates of local and systemic reactogenicity, which were greater than those seen for the placebo arm of the trials (Table 2 in Supplementary material). This reactogenicity, resulting from the inflammatory response to vaccination, tended to be greater with the second dose (when applicable), and it was less frequent and less severe in older recipients. Nearly all reactions to the vaccines were considered mild to moderate, and these reactions resolved within several days. Serious adverse events were similar in the vaccine and placebo arms, and no deaths considered attributable to vaccination were reported in any of the trials. ${ }^{27-29}$ Each of the manufacturers will continue safety monitoring of participants for up to 2 years.

\section{Postauthorization safety monitoring}

Knowing that even large clinical trials cannot detect rare adverse events, FDA and CDC are conducting extensive postauthorization safety monitoring. The CDC ACIP convened the Vaccine Safety Technical (VaST) subgroup to review, evaluate, and interpret postauthorization vaccine safety data and to serve as the central hub where technical subject matter experts from federal agencies can share vaccine safety data. Vaccine safety surveillance typically has relied on passive surveillance (ie, clinicians or even patients reporting adverse events after vaccination), primarily via the Vaccine Adverse Events Reporting System (VAERS), which is comanaged by CDC and FDA. To enhance this reporting system, CDC created v-safe, a smartphone-based software application that functions as a postvaccination health checker. All COVID-19 vaccine providers were asked to provide enrollment information to all recipients and to encourage recipients to register at the time of their vaccination. This system allows the assessment of vaccine side effects of all severities. V-safe then contacts those reporting serious adverse events and facilitate VAERS reporting, if appropriate. The safety monitoring systems in place are summarized in Table 3 (see Supplementary material). Ultimately, these combined systems allow for early safety signal detection, followed by rapid cycle analysis and case evaluations, as well as the ability to analyze large linked databases to further evaluate potential safety concerns in US populations.

Reported rates of expected side effects after receiving the PfizerBioNTech, Moderna, or J\&J/Janssen COVID-19 vaccine have been similar to those reported in clinical trials. ${ }^{30}$ Several unanticipated side effects have been identified through postauthorization monitoring. As new data emerge regarding the durability of COVID-19 vaccine response, effectiveness, and safety in special populations (eg, pregnant people, immunocompromised persons, etc), and the spread of new SARS-CoV-2 variants, the ACIP will review and revise COVID-19 vaccine information.

\section{Anaphylaxis}

Although no anaphylaxis reaction occurred in the clinical trials, anecdotal anaphylaxis or anaphylactoid reactions were reported shortly after EUAs were granted for the mRNA vaccines. Further analysis quantified the rate of anaphylaxis at $\sim 11$ per $1,000,000$ vaccine recipients for the Pfizer-BioNTech vaccine and 2.5 per 1,000,000 vaccine recipients for the Moderna vaccine, ${ }^{31-34}$ although higher rates have been reported via active surveillance. ${ }^{35}$ In these cases, anaphylaxis had an onset of $<30$ minutes in up to $90 \%$ of cases, and at least $80 \%$ of individuals with anaphylaxis had a documented allergy to drugs, medical products, food, or insect stings. ${ }^{31,34}$ Notably, up to $30 \%$ of the general population has a documented allergy to a food or medication, suggesting that 
anaphylaxis to an mRNA vaccine remains rare even in individuals with a history of allergy. ${ }^{31}$ Anaphylactic reactions were reported after the J\&J/Janssen vaccine in $<0.5$ per $1,000,000$ vaccine recipients. ${ }^{36}$ For comparison, after most vaccines, anaphylaxis is estimated to occur at a rate of $\sim 1.31$ per $1,000,000$ vaccine doses $(95 \% \mathrm{CI}, 0.90-1.84) .^{37}$

\section{$\mathrm{J} \& \mathrm{~J} / \mathrm{Janssen}$ and thrombosis with thrombocytopenia syndrome} In April 2021, CDC and FDA paused administration of the J\&J/ Janssen vaccine due to reports of unusual clotting events now known as thrombosis with thrombocytopenia syndrome (TTS). ${ }^{36}$ Similar cases had been reported previously with the AstraZeneca COVID-19 vaccine, another adenovirus vector vaccine not currently authorized for use in the United States. ${ }^{38,39}$ Patients presented with either venous thromboembolic disease or unusual thrombotic events associated with thrombocytopenia, such as cerebral venous sinus thrombosis, splanchnic vein thrombosis, hepatic vein thrombosis, or splenic vein thrombosis. ${ }^{36,40}$ Most of these cases were detected in women aged $<50$ years. In women aged 18-49 years, the incidence was 7 per 1,000,000 vaccine doses; in men aged 18-49 years, the incidence was 1 per 1,000,000 vaccine doses. ${ }^{41}$ The combination of these rare clotting events with thrombocytopenia is notable, and it suggests a mechanism similar to heparin-induced antiplatelet autoantibodies. ${ }^{38} \mathrm{FDA}$ and $\mathrm{CDC}$ called for vaccine administration to resume without age or gender restrictions after a 10-day pause in April 2021. ${ }^{41}$

\section{Myocarditis and pericarditis}

In May 2021, CDC issued a health advisory to inform vaccine providers and clinicians of a recent increase in reports of myocarditis and/or pericarditis after mRNA COVID-19 vaccination received in the VAERS. ${ }^{42}$ Most cases were reported in adolescent and young adult males within 4 days after the second dose, and these cases resolved without known sequelae. Israel's Health Ministry reported 275 cases of myocarditis after the Pfizer-BioNTech vaccine between December 2020 and May 2021, among $>5$ million vaccinated people. ${ }^{43}$ Similar to the US reports, most cases were in men aged 16-30 years and were mild. ${ }^{44-46}$ The investigation of the relationship between mRNA vaccines and myocarditis and pericarditis continues. CDC and the American Academy of Pediatrics (AAP) continue to recommend COVID-19 vaccination of adolescents and young men. ${ }^{42,44}$

\section{Safety in pregnancy and lactation}

Pregnant and lactating people were not included in the vaccine trials, but they were not prohibited from receiving the vaccines once they were authorized for emergency use. Since the EUA, the safety of the mRNA vaccines in pregnancy has been followed using the $\mathrm{v}$-safe pregnancy registry. ${ }^{1}$ Adverse events after vaccination were similar between pregnant and nonpregnant recipients, with more injection-site pain reported among pregnant recipients and more systemic reactions reported among nonpregnant recipients. ${ }^{30,47}$ Pregnancy and neonatal outcomes were reported in a subset of the v-safe registry. Rates of pregnancy loss (12.6\%) and stillbirth $(0.1 \%)$ were similar to published rates in the population $(10 \%-$ $26 \%$ and $<1 \%$ respectively). ${ }^{47}$ Rates of preterm birth $(9.4 \%)$ and infants who were small for gestational age (3.2\%) were also similar to those in the published literature. ${ }^{47}$ Safety of the J\&J/Janssen vaccine in pregnancy and lactation has not been studied to date. ${ }^{4-50}$ The American College of Obstetricians and Gynecologists (ACOG) and the Society for Maternal-Fetal Medicine (SMFM) recommend that COVID-19 vaccination be offered to pregnant and lactating women. ${ }^{48}$

\section{Dispelling vaccine myths}

Because of the rapid development and deployment of the COVID-19 vaccines, some of which use technologies not previously employed for vaccines licensed in the United States, a number of novel vaccine myths have circulated, including those regarding concerns about DNA integration and infertility. In addition, many people remain hesitant due to concerns about long-term side effects of vaccination. Although we must be mindful that long-term side effects will remain unknown until sufficient time has elapsed to assess for such possible effects, it is important to recognize that, among other vaccines that have had adverse events historically, nearly all side effects occurred within 6-8 weeks of vaccination. ${ }^{51}$ Communication guides and handbooks are available to assist vaccine program planners regarding how best to communicate to debunk myths and encourage vaccination. ${ }^{52-55}$

\section{Benefits of a fully vaccinated workforce}

Vaccination can be considered an elimination strategy in the framework of the Hierarchy of Controls. ${ }^{56}$ As such, it is expected to be one of the most effective interventions to reduce the risk of transmission in all settings (Fig. 1 in Supplementary material).

For routine vaccinations, and specifically for COVID-19 vaccines, the benefits of a fully vaccinated workforce can be categorized broadly into (1) reducing the risk of transmission within healthcare facilities among HCP and patients, from the community to healthcare facilities, and from healthcare facilities to the community; (2) maintaining a healthy workforce and supporting HCP wellness; and (3) maintaining the trustworthiness of HCP and healthcare institutions (Table 4 in Supplementary material).

\section{Improving coverage without vaccination as a condition of employment}

Some healthcare facilities have achieved high rates of HCP compliance with routinely recommended vaccines in the absence of vaccination as a condition of employment. A combination of strategies is more effective than a single strategy ${ }^{57,58}$; Table 5 (see Supplementary material) describes typical strategies. Notably, most programs reporting immunization rates of $90 \%$ or higher used 1 or more soft mandates, including requiring declination forms or the use of face masks by unvaccinated HCP during close contact with patients. Current universal masking by HCP as part of COVID-19 pandemic precautions precludes the use of a "vaccinate or mask" strategy to promote COVID- 19 vaccination. State statutes may positively affect HCP COVID-19 vaccination rates, as demonstrated by examples specific to influenza vaccination of HCP in the absence of facility requirements. ${ }^{59}$ Laws requiring hospitals to assess HCP influenza immunization status or to offer vaccination to HCP have also increased facility immunization rates. ${ }^{59}$

\section{Incentives}

Many healthcare facilities and systems, with and without vaccination as a condition of employment, have used incentives to encourage staff to receive recommended vaccines. ${ }^{60-62}$ According to Equal Employment Opportunity Commission (EEOC) guidance, employers may offer noncoercive incentives for voluntarily providing proof of vaccination or for receiving the vaccination itself. ${ }^{63}$ Although incentives generally are not permitted for receipt of federally funded services such as Medicaid or Medicare, most facilities 
provide vaccines free of charge to their employees and do not bill Medicaid or Medicare for them, so these rules do not appear to apply to employee incentive programs.

These considerations apply to all healthcare employers in the country, public and private. Employers must also consult with their attorneys to make sure they are complying with any state laws and local ordinances or orders specific to their location and public or private status. Although incentives have been successful in some centers to boost immunization rates (Table 5 in Supplementary material), their use may be subject to certain limitations when applied to increasing uptake of COVID-19 vaccines.

\section{Advantages of vaccination as a condition of employment}

The experience to date with voluntary influenza vaccination, as opposed to influenza vaccination as a condition of employment, suggests that without requiring COVID-19 vaccination, target coverage will rarely be achieved. ${ }^{64}$ In the most recent season for which data are available, $80.6 \%$ of HCP reported receiving influenza vaccination during the 2019-2020 season. ${ }^{65}$ Compliance among those who were required by their employer to receive the vaccination was $94.4 \%$, compared to $69.6 \%$ among those without vaccination as a condition of employment. ${ }^{66}$ Although vaccination is one of the most effective strategies to mitigate risk of transmission of communicable diseases, vaccination of HCP with ACIP-recommended vaccines prior to the COVID-19 pandemic has been suboptimal, with $~ 50 \%$ of surveyed HCP in March 2021 remaining unvaccinated. ${ }^{67}$ The National Vaccine Advisory Committee has recommended that employers consider requirements if their facilities are unable to achieve the Healthy People goal of at least $90 \%$ of HCP vaccinated for influenza. ${ }^{64}$ In 2020, SHEA recommended that medical contraindications should be accepted as a reason for not receiving all routine immunizations recommended by CDC. ${ }^{68}$ Federal statute Title VII $^{\mathrm{c}}$ regulates exemption from a vaccination policy on the basis of religious objection. Exemption requests should be evaluated by the appropriate department (eg, human resources and/or legal) on a case-by-case basis. ${ }^{63}$

\section{Legal considerations}

There is long-standing constitutional support for vaccine requirements, and many state laws also support them. According to EEOC published guidance, the federal civil rights laws it enforces do not prevent an employer from requiring employees to be vaccinated for COVID-19, subject to a limited set of legally required exceptions (medical contraindication based on CDC and manufacturer guidelines, disability, and religion). ${ }^{63}$ This section reviews key issues that a healthcare employer should discuss with legal counsel before making vaccination a condition of employment.

Legal debate has surrounded the fact that the COVID-19 vaccines are currently approved for use under an FDA EUA. The EEOC has referred employers to the FDA's posted guidance concerning EUAs. FDA promulgates the regulatory scheme governing EUAs. As of June 2021, only 1 trial court decision has addressed a legal challenge to an employee vaccination requirement on the basis of the EUA regulation. Although the court concluded that the regulation does not prohibit a private hospital system from requiring that its employees receive vaccines under an EUA as a condition of employment, it does not establish a precedent. $^{\mathrm{d}}$

${ }^{c} 42$ U.S.C. $\$ 2000 \mathrm{e}-2$ (Title VII) is the federal statute prohibiting discrimination on the basis of religion, and regulates the employer's treatment of employees as "individuals."

${ }^{\mathrm{d}}$ See Bridges v. Houston Methodist Hosp., No. CV H-21-1774, 2021 WL 2399994, at *2 (S.D. Tex. Jun. 12, 2021). A private hospital employer required all employees to receive the
The regulation by its terms requires FDA to establish the conditions under which an EUA product is administered by the medical provider, including by obtaining the recipient's informed consent, but there is no mention of employers or employment policies in the regulation, including requirements of employers. ${ }^{e}$ FDA guidance concerning the COVID-19 vaccines also makes no mention of employees; rather, it states that the required information concerning an EUA product is typically communicated to the recipient in a "patient fact sheet" that FDA makes available on its website. ${ }^{69}$ The text of the EUA regulation does not, in other words, require that individuals electing to receive a product approved under EUA undergo the informed consent process required for participation in clinical trials. The debate concerning the EUA status of the COVID-19 vaccines highlights the distinction between a healthcare organization's role as medical provider versus its role as employer. An employer's requirement that its employees be vaccinated as a condition of employment is distinct from requiring them to be vaccinated without consent; that is, an individual has a right to refuse vaccination but has no right to a particular job. In at-will employment, an employee may always pursue alternative employment if they do not wish to be vaccinated as a condition of employment. The same is true of a condition of employment that is established in a unionized environment, although the employer should review its collective bargaining agreement, including, for example, the clauses concerning management rights, health and safety, and exigent circumstances, to determine whether bargaining is or is not required before establishing a condition of employment. At the same time, to try to avoid consequences, such as the loss of talented team members in whom employers have invested time and money or a decrease in employee morale or engagement, employers should take steps to address employee concerns regarding safety, efficacy, equity, and inclusion.

\section{Federal and state rules}

Any employer's requirement of vaccination as a condition of employment will be subject to federal employment laws, namely the Americans with Disabilities Act (ADA) and Title VII of the Civil Rights Act of $1964 .{ }^{63}$ As relevant to the vaccine context, these statutes prohibit employers from discriminating against their employees on the basis of disability or religion, and they require employers to provide reasonable accommodations to any employee with a disability or religious objection. A reasonable accommodation is decided by the employer on a case-by-case basis and does not include measures that would result in undue hardship or significant difficulty or expense for the employer or measures that pose a direct threat to the health and safety of others.

COVID-19 vaccination by June 7, 2021, federal district court in Texas ruled that the EUA regulation confers certain powers to the Secretary of Health and Human Services in an emergency, without affecting the responsibilities of the private employer or conferring a right of private action to the employees. The regulation furthermore does not require employees to participate in a human trial, but only requires that informed consent be obtained from the recipient of the vaccine before administering it.

eThe informed consent provision in the EUA regulation reads in full: "With respect to emergency use of an unapproved product, the $[\mathrm{FDA}]$, to the extent practicable given the applicable circumstances ... shall, for a person who carries out any activity for which the authorization is issued, establish such conditions on an [EUA] as the [FDA] finds necessary and appropriate to protect public health, including the following: ... (ii) Appropriate conditions designed to ensure that individuals to whom the product is administered are informed - (I) that the [FDA] has authorized the emergency use of the product; (II) of the significant known and potential benefits and risks of such use, and of the extent to which such benefits and risks are unknown; and (III) of the option to accept or refuse administration of the product, of the consequences, if any, of refusing administration of the product, and of the alternatives to the product that are available and of their benefits and risks.” (21 U.S.C. $\$ 360$ bbb-3(e)(1)(A)(ii).) 
Although these federal laws set the baseline for all employers, state laws may impose additional requirements.

The standards imposed by OSHA are also relevant to any employer considering a policy of vaccination as a condition of employment. OSHA recently published an Emergency Temporary Standard that addresses COVID-19 vaccination but without explicitly endorsing or prohibiting employer requirements. The standard requires employers to "support" COVID19 vaccination for their employees by providing them with paid time off to receive the vaccine and to recover from associated side effects. ${ }^{3}$ The standard also suggests support for a vaccinated workforce. For example, it does not extend to "fully vaccinated" ambulatory or home healthcare settings, and it does not require vaccinated $\mathrm{HCP}$ who have been exposed to the virus to be removed from work.

\section{Exemptions}

Critical to the success of any vaccine requirement is a clear and consistent process by which employers receive, review, and respond to the exemption and accommodation requests that are required by the ADA, Title VII, and parallel state employment discrimination laws. If an exemption is granted, an employer may require employees to comply with accommodations in lieu of receiving the vaccine. Accommodations may include providing an alternative form of the vaccine, requiring an exempted employee to wear a face mask, or requiring an exempted employee to follow physical distancing measures (including reassignment away from vulnerable patient populations, curtailing job duties to lessen or eliminate direct patient contact, or allowing the employee to work remotely if feasible). The law requires that accommodations be tailored to the individual employee and their particular job duties; for that reason, employers should try to avoid making blanket statements about what they will or will not do if an employee qualifies as exempt from a vaccination requirement. To mitigate the risk of liability based on alleged retaliatory or discriminatory denials of such requests, and to maintain confidentiality, medical exemption and accommodation requests are usually reviewed by occupational health staff, and religious requests are commonly routed to the employer's legal and/or human resources department(s) for review. Those departments also typically keep records of what accommodations have been requested, considered, and discussed with the employee, and whether they are granted or rejected. They may have an appeal process. Employers often require the qualifying employee to read and sign their acknowledgement of the accommodation plan, which may include a statement of the risks of remaining unvaccinated.

\section{Religious exemption}

For religious exemption requests, an employer should consider making a form available for objectors to describe their sincerely held religious belief, practice, or observance (whether connected to a traditionally recognized religion or held with the strength of traditional religious views) on which basis they seek an exemption from vaccination. Although affiliation with a traditionally organized religion may be evidence to support a claim of a sincerely held

\footnotetext{
${ }^{f}$ The federal statutes prohibiting discrimination on the basis of disability (ADA) and religion (Title VII). Both regulate the employer's treatment of employees as "individuals." See 42 U.S.C. $\$ 12102(2)$, (2)(A) (ADA); 42 U.S.C. $\$ 2000$ e- 2 (Title VII); see also, for example, Albertson v. Kirkingburg, 527 U.S. 555, 566. The US Supreme Court explains that the ADA, by its terms, applies to "individuals" and therefore imposes statutory obligations that must be assessed on a "case-by-case basis."
}

religious belief, the lack of such an affiliation cannot be the basis for rejecting an exemption request.

\section{Medical exemptions}

A medical exemption is based on contraindications and precautions set forth by the manufacturer or $\mathrm{CDC}$ and usually requires review and signature by a medical professional. ${ }^{70}$ Many organizations also will allow for deferrals during pregnancy, if requested, or for other time-limited conditions upon request. Medical exemption request submissions citing other reasons for exemption should be permitted and reviewed for special circumstances. The substantive basis of all requests for an exemption based on a medical contraindication or precaution are ideally reviewed by an organization's occupational health medical director or committee of clinicians.

\section{Enforcement}

Some employees may not qualify for any of the employers' exemptions and may decline to be vaccinated. The consequences of noncompliance with a vaccination policy as a condition of employment should be clearly defined and understood by leadership, the legal team, and human resources department(s) before the policy is enacted, and it should be clearly communicated to employees. Enforcement mechanisms may include, for example, letters of warning, suspension without pay, or termination. For facilities or systems with unionized employees, it is important to engage union representatives early in this process. ${ }^{71,72}$

\section{Privacy concerns}

Some employees may object to providing information about their vaccination status on privacy grounds. In its COVID-19 guidance, the EEOC has stated that an employer may, without violating the applicable federal laws, ask an employee about their vaccination status and require proof of vaccination as long as it is stored as confidential medical information.

\section{Visual cues}

Healthcare employers may use visual cues, such as stickers, to indicate work authorization and work restrictions appropriate to an employee's vaccination status, provided that any visual cues do not make explicit reference to whether or not the employee has been vaccinated. ${ }^{g}$ With or without the use of visual cues, employers with policies of vaccination as a condition of employment should clearly communicate to and remind employees that harassment or retaliatory behavior against coworkers is never tolerated, including with regard to suspected COVID-19 vaccination status. Before implementing a COVID-19 vaccination policy that utilizes visual cues, facilities should plan for what they will do if a patient or family member suspects that an HCP is unvaccinated and refuses care. These plans should include communications protocols that explain which precautions are in place to protect them. Facilities should also communicate clearly that unvaccinated persons do not need to disclose the reason for not being vaccinated to other HCP or to patients.

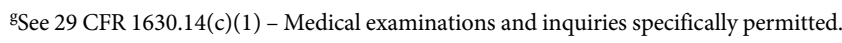




\section{Implementing COVID-19 vaccine as a condition of employment}

Healthcare facilities face a complex and strategic decision regarding COVID-19 vaccine as a condition of employment; it requires consideration of a healthcare facility's mission and culture. Transparency by leadership during the decision process garners trust and credibility among staff and medical staff, as does an inclusive approach that reflects the diversity of opinion and backgrounds of HCP, while visibly engaging stakeholders and facilitating input. Leaders must assess perceptions among HCP and uphold principles of respect and inclusivity that reflect diversity of opinion and background.

If adopted, operationalizing a condition of employment requires facilities to have systems in place for tracking healthcare personnel vaccination status both at the facility and offsite locations, establishing policies and processes for exemption request and review, and addressing important topics such as equity, compensation for vaccination, and postvaccination sick days.

In a recent policy brief, the WHO recommended that local context be considered to determine whether a mandate is necessary, proportionate, and would not undermine trust. ${ }^{73}$ Although not likely to influence the requirement itself, facilities should acknowledge issues at the organizational and individual levels to ensure smooth implementation. Many of these issues are addressed throughout this document, including safety and efficacy, institutional culture of respect and inclusivity, consistent and transparent exemption review, and collaboration with unions and other stakeholders. A framework for implementation is provided in a companion implementation guide.

Acknowledgments. We acknowledge Lauri Hicks, DO, for sharing her expertise to help with the development of this document, and Armand H. Matheny Antommaria, $\mathrm{MD}, \mathrm{PhD}$ for his expert review. The findings and conclusions in this document are those of the authors, who are responsible for its content, and they do not necessarily represent the views of their institutions, the Veterans' Administration, or the US government. The authors thank Valerie Deloney, MBA, for her organizational expertise in the development of this manuscript.

Financial support. This work was supported in part by funds and facilities provided by the Geriatric Research Education and Clinical Center (GRECC) at the VA Northeast Ohio Healthcare System, Cleveland, Ohio.

Conflicts of interest. The following disclosures are a reflection of what has been reported to SHEA. To provide thorough transparency, SHEA requires full disclosure of all relationships, regardless of relevancy to the guideline topic. Such relationships as potential conflicts of interest are evaluated in a review process that may include the Board of Trustees and Editor of Infection Control and Hospital Epidemiology. The assessment of disclosed relationships for possible conflicts of interest has been based on the relative weight of the financial relationship (ie, monetary amount) and the relevance of the relationship (ie, the degree to which an association might reasonably be interpreted by an independent observer as related to the topic or recommendation of consideration). D.J.W. is a consultant for Merck (vaccines), Pfizer (vaccines), PDI, Germitec, and UV Innovators. H.M.B. receives research funding with CDC Epicenter COVID-19 supplement: SHEPHERD COVID-19 Vaccine Effectiveness. K.B. is a clinical investigator on vaccine trials funded by Pfizer (pneumococcal vaccine and COVID-19 vaccines), Gilead, and Enanta, and receives honoraria from WebMed for a column in Pediatric News. R.E. is a coinvestigator on a study supported by Thermo Fisher examining the role of procalcitonin-supported antimicrobial stewardship efforts on antibiotic utilization. R.L.P.J. has research grants from Pfizer, Merck, and previously from Accelerate for projects related to bacterial infections, drug-resistant bacteria, and antibiotic stewardship, is on advisory boards for Pfizer for topics related to immunizations against against bacterial infections, and is on an advisory board for Pfizer with regard to their COVID-19 vaccine that was specific to the physical packaging and distribution of the vaccine (cold chain, dry ice, etc).
F.L. is a clinical primary investigator for a CDC-funded small business development grant: Multiplex Detection of MDROs and Lower Intestinal Microbiome Disruption. A.N.M. has a research grant from CDC for the US Hospital Vaccine Effectiveness Network (grant no. 5 U01IP000974-05) to assess influenza vaccine effectiveness in preventing influenza-related hospitalizations. T.A.M. received an honorarium for a March 2021 grand rounds/CME lecture on COVID-19 vaccines delivered to faculty and trainees at a hospital in Michigan. Z.W. is the Emory University Hospital Midtown Site Lead Investigator, with research grants from Kinevant and the National Institutes of Health for DMID Protocol 20-0006: A Multicenter, Adaptive, Randomized Blinded Controlled Trial of the Safety and Efficacy of Investigational Therapeutics for the Treatment of COVID-19 in Hospitalized Adults. Z.W. is also a subinvestigator in the A Multicenter, Adaptive, Randomized, Double-blind, Placebo-controlled Study to Assess the Efficacy and Safety of Gimsilumab in Subjects With Acute Lung Injury or Acute Respiratory Distress Syndrome (ARDS) Secondary to Coronavirus Disease. D.Y. is included as an investigator on CDC (grant no. U54 CK000484) Prevention Epicenters IV but does not receive any personal financial support. H.Y. has a research grant from NIH for ACCT trials for COVID-19 treatment. All other authors report no conflicts of interest related to this article.

Supplementary material. To view supplementary material for this article, please visit https://doi.org/10.1017/ice.2021.322

\section{References}

1. CDC, NCIRD. v-safe COVID-19 vaccine pregnancy registry 2021. Centers for Disease Control and Prevention website. https://www.cdc.gov/ coronavirus/2019-ncov/vaccines/safety/vsafepregnancyregistry.html. Updated June 16, 2021. Accessed July 12, 2021.

2. CDC, NCIRD. Pregnant and recently pregnant people at increased risk for severe ILLNESS from COVID-19. Centers for Disease Control and Prevention website. https://www.cdc.gov/coronavirus/2019-ncov/needextra-precautions/pregnant-people.html. Updated June 10, 2021. Accessed June 2021.

3. OSHA. Occupational exposure to COVID-19; Emergency Temporary Standard. June 21, 2021 ed. Federal Register. p. 32376-628.

4. Office of Disease Prevention and Health Promotion. Topics \& objectives: immunization and infectious diseases. HealthyPeople.gov website. https:// www.healthypeople.gov/2020/topics-objectives/topic/immunization-andinfectious-diseases/objectives. Published 2020. Accessed July 12, 2021.

5. Coronavirus in the United States: latest map and case count. New York Times May 13, 2021.

6. Kalish H, Klumpp-Thomas C, Hunsberger S, et al. Undiagnosed SARSCoV-2 seropositivity during the first six months of the COVID-19 pandemic in the United States. Sci Transl Med 2021. doi: 10.1126/ scitranslmed.abh3826.

7. Vaccine development - 101. US Food and Drug Administration website. https://www.fda.gov/vaccines-blood-biologics/development-approval-processcber/vaccine-development-101. Updated December 14, 2020. Accessed July 12, 2021.

8. Emergency use authorization. US Food and Drug Administration website. https://www.fda.gov/emergency-preparedness-and-response/mcm-legalregulatory-and-policy-framework/emergency-use-authorization. Updated June 28, 2021. Accessed July 12, 2021.

9. Pfizer and BioNTech initiate rolling submission of biologics license application for US FDA approval of their COVID 19 vaccine. Pfizer website. https://www.pfizer.com/news/press-release/press-release-detail/pfizerand-biontech-initiate-rolling-submission-biologics. Published May 7, 2021. Accessed June 2021.

10. Moderna announces FDA authorization of Moderna COVID-19 vaccine. Moderna website. https://investors.modernatx.com/news-releases/newsrelease-details/moderna-announces-fda-authorization-moderna-covid-19vaccine-us. Updated December 18, 2020. Accessed June 2021.

11. Landscape of COVID-19 candidate vaccines. World Health Organization website. https://www.who.int/publications/m/item/draft-landscape-of-covid19-candidate-vaccines. Updated May 11, 2021. Accessed July 12, 2021. 
12. Perl TM, Talbot TR. Universal influenza vaccination among healthcare personnel: yes we should. Open Forum Infect Dis 2019;6(4):ofz096.

13. Polack FP, Thomas SJ, Kitchin N, et al. Safety and efficacy of the BNT162b2 mRNA COVID-19 vaccine. N Engl J Med 2020;383:2603-2615.

14. Sadoff J, Gray G, Vandebosch A, et al. Safety and efficacy of single-dose Ad26.COV2.S vaccine against COVID-19. N Engl J Med 2021;384:2187-2201.

15. Shroff RT, Chalasani P, Wei R, et al. Immune responses to COVID-19 mRNA vaccines in patients with solid tumors on active, immunosuppressive cancer therapy. medRxiv 2021. doi: 10.1101/2021.05.13.21257129.

16. Chodick G, Tene L, Rotem RS, et al. The effectiveness of the two-dose BNT162b2 vaccine: analysis of real-world data. Clin Infect Dis 2021. doi: 10.1093/cid/ciab438.

17. Deepak P, Kim W, Paley MA, et al. Glucocorticoids and B-cell-depleting agents substantially impair immunogenicity of mRNA vaccines to SARSCoV-2. medRxiv. 2021. doi: 10.1101/2021.04.05.21254656.

18. Levine-Tiefenbrun M, Yelin I, Katz R, et al. Initial report of decreased SARS$\mathrm{CoV}-2$ viral load after inoculation with the BNT162b2 vaccine. Nat Med 2021;27:790-792.

19. Teran RA, Walblay KA, Shane EL, et al. Postvaccination SARS-CoV-2 infections among skilled nursing facility residents and staff members-Chicago, Illinois, December 2020-March 2021. Morb Mortal Wkly Rep 2021; 70:632-638.

20. Benenson S, Oster Y, Cohen MJ, Nir-Paz R. BNT162b2 mRNA COVID-19 vaccine effectiveness among healthcare workers. N Engl J Med 2021; 384:1775-1777.

21. Amit S, Regev-Yochay G, Afek A, Kreiss Y, Leshem E. Early rate reductions of SARS-CoV-2 infection and COVID-19 in BNT162b2 vaccine recipients. Lancet 2021;397:875-877.

22. Hughes MM, Groenewold MR, Lessem SE, et al. Update: characteristics of healthcare personnel with COVID-19-United States, February 12-July 16, 2020. Morb Mortal Wkly Rep 2020;69:1364-1368.

23. Edridge AWD, Kaczorowska J, Hoste ACR, et al. Seasonal coronavirus protective immunity is short-lasting. Nat Med 2020;26:1691-1693.

24. CNBC News. Pfizer CEO says third COVID vaccine dose likely needed within 12 months. CNBC website. https://www.cnbc.com/2021/04/15/pfizer-ceo-saysthird-covid-vaccine-dose-likely-needed-within-12-months.html. Published April 2021. Accessed July 12, 2021.

25. CBS News. Moderna plans to have third vaccine booster shot ready by fall 2021. CBS News website. https://www.cbsnews.com/news/moderna-covidvaccine-booster-shots/. Published April 19, 2021. Accessed July 12, 2021.

26. CNN News. Johnson \& Johnson working on booster for coronavirus variants, CEO says CNN website. https://edition.cnn.com/world/live-news/coronaviruspandemic-vaccine-updates-03-01-21/h_a3a215720177e6958995c55169 a94c65. Accessed May 13, 2021.

27. Polack FP, Thomas SJ, Kitchin N, et al. Safety and efficacy of the BNT162b2 mRNA COVID-19 vaccine. N Engl J Med 2020;383:2603-2615.

28. Baden LR, El Sahly HM, Essink B, et al. Efficacy and safety of the mRNA1273 SARS-CoV-2 vaccine. N Engl J Med 2021;384:403-416.

29. Sadoff J, Gray G, Vandebosch A, et al. Safety and efficacy of single-dose Ad26.COV2.S vaccine against COVID-19. N Engl J Med 2021. doi: 10.1056/NEJMoa2101544.

30. Gee J, Marquez P, Su J, et al. First month of COVID-19 vaccine safety monitoring-United States, December 14, 2020-January 13, 2021. Morb Mortal Wkly Rep 2021;70:283-288.

31. Team CC-R, Administration FaD. Allergic reactions including anaphylaxis after receipt of the first dose of Moderna COVID-19 vaccine-United States, December 21, 2020-January 10, 2021. Morb Mortal Wkly Rep 2021;70:125-129.

32. Castells MC, Phillips EJ. Maintaining safety with SARS-CoV-2 vaccines. $N$ Engl J Med 2021;384:643-649.

33. Shimabukuro TT, Cole M, Su JR. Reports of anaphylaxis after receipt of mRNA COVID-19 vaccines in the United States-December 14, 2020January 18, 2021. JAMA 2021;325:1101-1102.

34. CDC COVID-19 Response Team; Food and Drug Administration. Allergic reactions including anaphylaxis after receipt of the first dose of PfizerBioNTech COVID-19 vaccine-United States, December 14-23, 2020. Morb Mortal Wkly Rep 2021;70:46-51.
35. Blumenthal KG, Robinson LB, Camargo CA, et al. Acute allergic reactions to mRNA COVID-19 vaccines. JAMA 2021;325:1562-1565.

36. Shay DK, Gee J, Su JR, et al. Safety monitoring of the Janssen (Johnson \& Johnson) COVID-19 vaccine-United States, March-April 2021. Morb Mortal Wkly Rep 2021;70:680-684.

37. McNeil MM, Weintraub ES, Duffy J, et al. Risk of anaphylaxis after vaccination in children and adults. J Allergy Clin Immunol 2016;137:868-878.

38. Greinacher A, Thiele T, Warkentin TE, Weisser K, Kyrle PA, Eichinger S. Thrombotic thrombocytopenia after ChAdOx1 nCov-19 vaccination. N Engl J Med 2021;384:2092-2101.

39. Schultz NH, Sørvoll IH, Michelsen AE, et al. Thrombosis and thrombocytopenia after ChAdOx1 nCoV-19 vaccination. N Engl J Med 2021;384: 2124-2130.

40. Shimabukuro T, editor. Update: Thrombosis with thrombocytopenia syndrome (TTS) following COVID-19 vaccination. Centers for Disease Control and Prevention website. https://www.cdc.gov/vaccines/acip/meetings/ downloads/slides-2021-04-23/03-COVID-Shimabukuro-508.pdf. Published April 23, 2021. Accessed July 12, 2021.

41. MacNeil JR, Su JR, Broder KR, et al. Updated recommendations from the advisory committee on immunization practices for use of the Janssen (Johnson \& Johnson) COVID-19 vaccine after reports of thrombosis with thrombocytopenia syndrome among vaccine recipients - United States, April 2021. Morb Mortal Wkly Rep 2021;70:651-656.

42. CDC, NCIRD. Myocarditis and pericarditis following mRNA COVID-19 vaccination. Centers for Disease Control and Prevention website. https:// www.cdc.gov/coronavirus/2019-ncov/vaccines/safety/myocarditis.html. Published 2021. Accessed July 12, 2021.

43. Israel Ministry of Health. Surveillance of myocarditis (inflammation of the heart muscle) cases between December 2020 and May 2021. Israel Ministry of Health website. https://www.gov.il/en/departments/news/01062021-03. Published June 2, 2021. Accessed July 12, 2021.

44. Jenco M. Report details 7 cases of myocarditis after COVID-19 vaccination AAP Publications website. https://www.aappublications.org/news/2021/ 06/04/covid-vaccine-myocarditis-case-reports-060421. Published 2021. Accessed July 2021.

45. Heller J. Israel sees probable link between Pfizer vaccine and myocarditis cases 2021. Reuters website. https:/www.reuters.com/world/middle-east/israelsees-probable-link-between-pfizer-vaccine-small-number-myocarditiscases-2021-06-01/. Published June 1, 2021. Accessed July 12, 2021.

46. Myocarditis and pericarditis following mRNA COVID-19 vaccination. Centers for Disease Control and Prevention website. https://www.cdc. gov/coronavirus/2019-ncov/vaccines/safety/myocarditis.html. Updated June 23, 2021. Accessed July 12, 2021.

47. Shimabukuro TT, Kim SY, Myers TR, et al. Preliminary findings of mRNA COVID-19 vaccine safety in pregnant persons. N Engl J Med 2021;384: 2273-2282.

48. ACOG and SMFM joint statement on WHO recommendations regarding COVID-19 vaccines and pregnant individuals. American College of Obstetricians and Gynecologists website. https://www.acog.org/news/newsreleases/2021/01/acog-and-smfm-joint-statement-on-who-recommendationsregarding-covid-19-vaccines-and-pregnant-individuals. Published January 27, 2021. Accessed July 12, 2021.

49. Society for Maternal-Fetal Medicine (SMFM) Statement: SARS-CoV-2 vaccination in pregnancy. Society for Maternal-Fetal Medicine website. https:// www.smfm.org/publications/339-society-for-maternal-fetal-medicine-smfmstatement-sars-cov-2-vaccination-in-pregnancy. Published 2020. Accessed July 12, 2021.

50. Riley L, Beigi R, Jamieson D, et al. ACOG clinical practice advisory: COVID-19 vaccination considerations for obstetric-gynecologic care. American College of Obstetricians and Gynecologists website. https:// www.acog.org/clinical/clinical-guidance/practice-advisory/articles/2020/12/ covid-19-vaccination-considerations-for-obstetric-gynecologic-care?utm source=redirect\&utm_medium=web\&utm_campaign=int. Published 2020. Accessed June 2021.

51. Long-term side effects of COVID-19 vaccine. Children's Hospital of Philadelphia website. https://www.chop.edu/news/long-term-side-effectscovid-19-vaccine. Accessed June 2021. 
52. CDC, NCIRD. Vaccinate with confidence. Centers for Disease Control and Prevention website. https:/www.cdc.gov/vaccines/covid-19/vaccinate-withconfidence.html. Published 2021. Accessed June 2021.

53. Lewandowsky S, Cook J, Schmid P, Holford D, Finn A, Leask J. The COVID-19 vaccine communication handbook. A practical guide for improving vaccine communication and fighting misinformation. Michigan Medicine Office for Health Equity \& Inclusion website. https://ohei.med.umich.edu/sites/default/ files/files/downloads/FINAL\%20COVID19VaccineHandbook\%204\% 20production.pdf. Published 2021. Accessed July 12, 2021.

54. Vaccine misinformation management field guide. UNICEF website. https:// vaccinemisinformation.guide/. Accessed June 2021.

55. Yale Institute for Global Health: Vaccine messaging guide UNICEF website. https://www.unicef.org/media/93661/file/Vaccine messaging guide.pdf. Accessed June 2021.

56. CDC, NIOSH. Hierarchy of controls. Centers for Disease Control and Prevention website. https:/www.cdc.gov/niosh/topics/hierarchy/default. html. Published 2015. Accessed June 2021.

57. Rashid H, Yin JK, Ward K, King C, Seale H, Booy R. Assessing interventions to improve influenza vaccine uptake among healthcare workers. Health Aff (Millwood) 2016;35:284-292.

58. Lytras T, Kopsachilis F, Mouratidou E, Papamichail D, Bonovas S. Interventions to increase seasonal influenza vaccine coverage in healthcare workers: a systematic review and meta-regression analysis. Hum Vaccin Immunother 2016;12:671-681.

59. Lindley MC, Mu Y, Hoss A, et al. Association of state laws with influenza vaccination of hospital personnel. Am J Prev Med 2019;56:e177-e183.

60. Drees M, Wroten K, Smedley M, Mase T, Schwartz JS. Carrots and sticks: achieving high healthcare personnel influenza vaccination rates without a mandate. Infect Control Hosp Epidemiol 2015;36:717-724.

61. Podczervinski S, Stednick Z, Helbert L, et al. Employee influenza vaccination in a large cancer center with high baseline compliance rates: comparison of carrot versus stick approaches. Am J Infect Control 2015;43: 228-233.

62. Heinrich-Morrison K, McLellan S, McGinnes U, et al. An effective strategy for influenza vaccination of healthcare workers in Australia: experience at a large health service without a mandatory policy. BMC Infect Dis 2015;15:42.

63. What you should know about COVID-19 and the ADA, the Rehabilitation Act, and other EEO laws. Equal Employment Opportunity Commission website. https:/www.eeoc.gov/wysk/what-you-should-know-about-covid19-and-ada-rehabilitation-act-and-other-eeo-laws. Updated June 28, 2021. Accessed June 2021.
64. National Vaccine Advisory Committee. Strategies to achieve the healthy people 2020 annual influenza vaccine coverage goal for health-care personnel: recommendations from the national vaccine advisory committee. Public Health Rep 2013;128:7-25.

65. CDC, NCIRD. Influenza vaccination coverage among health care personnel — United States, 2019-2020 influenza season. Centers for Disease Control and Prevention website. https://www.cdc.gov/flu/fluvaxview/hcp-coverage_ 1920estimates.htm. Updated October 1, 2020. Accessed June 2021.

66. Acero $\mathrm{C}$, Razzaghi H, Black $\mathrm{C}$, et al. Influenza vaccination coverage among healthcare personnel-United States, 2019-2020 influenza season. Centers for Disease Control and Prevention website. https://www.cdc.gov/flu/ fluvaxview/hcp-coverage_1920estimates.htm. Published 2020. Accessed July $12,2021$.

67. Kirzinger A, Kearney A, Hamel L, Brodie M. KFF/The Washington Post frontline health care workers survey. Kaiser Family Foundation website. https://www.kff.org/coronavirus-covid-19/poll-finding/kff-washington-posthealth-care-workers/. Published 2021. Accessed July 12, 2021.

68. Weber DJ, Talbot TR, Weinmann A, et al. Policy statement from the Society for Healthcare Epidemiology of America (SHEA): Only medical contraindications should be accepted as a reason for not receiving all routine immunizations as recommended by the Centers for Disease Control and Prevention. Infect Control Hosp Epidemiol 2021;42:1-5.

69. Fact sheet for recipients and caregivers: emergency use authorization (EUA) of the Moderna COVID-19 vaccine to prevent coronavirus disease 2019 (COVID-19) in individuals 18 years of age and older. US Food \& Drug Administration website. https://www.fda.gov/media/146638/download. Updated March 26, 2021. Accessed July 12, 2021.

70. Frequently asked questions about COVID-19 vaccination. Centers for Disease Control and Prevention website. https://www.cdc.gov/ coronavirus/2019-ncov/vaccines/faq.html. Updated June 15, 2021. Accessed June 2021.

71. National Nurses United response to COVID-19. National Nurses United website https://www.nationalnursesunited.org/covid-19. Updated May 2021. Accessed June 2021.

72. Godar T, Potter T. Funny you should ask: is a vaccine mandate a subject of bargaining? Healthcare Law Insights website. https://www.health carelawinsights.com/2021/05/funny-you-should-ask-is-a-vaccine-mandatesubject-of-bargaining/. Published 2021. Accessed July 12, 2021.

73. COVID-19 and mandatory vaccination: ethical considerations and caveats: World Health Organization website. https://www.who.int/publications/i/ item/WHO-2019-nCoV-Policy-brief-Mandatory-vaccination-2021.1. Updated April 13, 2021. Accessed June 2021. 